\title{
Registro de ocorrência de Panstrongylus lignarius (Walker, 1837) (Hemiptera: Reduviidae: Triatominae) no Estado de Mato Grosso, Brasil
}

\author{
Aécio Moraes de PAULA ${ }^{1,2}$, Maria Ignez CASTRILLON ${ }^{2,3}$,Giovana Belém Moreira Lima MACIEL ${ }^{2}$, \\ Cleber GALVÃO ${ }^{4}$
}

\section{RESUMO}

Neste trabalho, relata-se pela primeira vez a ocorrência de Panstrongylus lignarius no Estado de Mato Grosso. Entre 2001 e 2009, cinco espécimes foram capturados pelos moradores dos municípios de Paranaíta, Alta Floresta, Lucas do Rio Verde, Sorriso e Guarantá do Norte e enviados ao Laboratório de Entomologia da Secretaria de Estado de Saúde de Mato Grosso, onde foram identificados como Panstrongylus lignarius (Walker, 1837). O encontro dessa espécie no estado do Mato Grosso, Brasil, amplia a sua distribuição geográfica.

PALAVRAS-CHAVE: doença de Chagas, barbeiros, nova ocorrência.

\section{New records of Panstrongylus lignarius (Walker, 1837) (Hemiptera: Reduviidae: Triatominae), in the state of Mato Grosso, Brazil}

\section{ABSTRACT}

In this paper is reported for the first time the occurrence of Panstrongylus lignarius in the State of Mato Grosso. From 2001 to 2009, five specimens were caught in the municipalities of Paranaíta, Alta Floresta, Lucas do Rio Verde, Sorriso and Guarantã do Norte, and sent to the Laboratório de Entomologia da Secretaria de Estado de Saúde de Mato Grosso where they were identified as Panstrongylus lignarius (Walker, 1837). The presence of this species in the state of Mato Grosso, Brazil expands its geographical distribution.

KEYWORDS: Chagas disease, kissing bug, new records

\footnotetext{
${ }^{1}$ Responsável técnico pelo Programa Estadual de Controle da Doença de Chagas do Estado de Mato Grosso. Av. Adauto Botelho s/ño Parque da Saúde, Bairro Coxipó da Ponte, CEP: 78.085-200, Cuiabá, MT, Brasil. Telefax: 5565 3661-2494. E-mail: aecipaul@gmail.com

2 Laboratório de Entomologia, Secretaria de Estado de Saúde de Mato Grosso, Cuiabá, MT, Brasil. E-mail: ignezcas@hotmail.com

${ }^{3}$ Departamento de Ciências Biológicas, Universidade do Estado de Mato Grosso (UNEMAT), Cáceres, MT, Brasil. E-mail: giovanabelem@gmail.com

${ }^{4}$ Laboratório Nacional e Internacional de Referencia em Taxonomia de Triatomíneos, Instituto Oswaldo Cruz, Fiocruz, Rio de Janeiro, RJ, Brasil. E-mail: clebergalvao@gmail.com
} 
O gênero Panstrongylus Berg, 1879 possui grande importância na transmissão vetorial da doença de Chagas. Suas espécies têm ampla distribuiçáo na região Neotropical e apresentam habitats e comportamentos variados, incluindo registros de invasão e colonização de moradias (Santos et al. 2003). O gênero está atualmente composto por 14 espécies: P. chinai (Del Ponte, 1929); P. diasi Pinto \& Lent, 1946; P. geniculatus (Latreille, 1811); P. guentheri Berg, 1879; P. howardi (Neiva, 1911); P. humeralis (Usinger, 1939); P. lenti Galvão \& Palma, 1968; P. lignarius (Walker, 1873); P. lutzi (Neiva \& Pinto, 1923); P. martinezorum Ayala, 2009; P. megistus (Burmeister, 1835); P. mitarakaensis Bérenger \& Blanchet, 2007; P. rufotuberculatus (Champion, 1899); e P. tupynambai Lent, 1942. Destas, dez já foram encontradas no Brasil, onde $P$. megistus e P. geniculatus são as mais amplamente distribuídas. No estado do Mato Grosso apenas P. megistus, P. geniculatus e $P$. rufotuberculatus haviam sido registradas. Carcavallo $e t$ al. (1999) incluíram no complexo P. lignarius, três espécies de grande semelhança morfológica e cromática: P. lignarius, P. humeralis e P. herreri. Em 2002 Marcilla et al. (2002) por meio de análises de DNA ribossomal demonstraram que $P$. lignarius e $P$. herreri são uma mesma espécie e propuseram sinonimizá-las, no ano seguinte, ao publicarem a lista de espécies de triatomíneos válidas Galvão et al.(2003) aceitaram a sinonímia de P. herreri Wygodzinsky, 1948 com P. lignarius. Deste modo, o complexo P. lignarius ficou com duas espécies, P. humeralis restrita à República do Panamá e P. lignarius com distribuição na América do Sul. Morfologicamente são facilmente distinguíveis, pois, $P$. lignarius apresenta escutelo parcialmente preto com faixa amarela mediana longitudinal, processo posterior do escutelo com leve corcova basal, lobo anterior do pronoto com tubérculos sublaterais e tíbias com pequena mancha sub-basal amarela; enquanto $P$. humeralis possui escutelo amarelado com faixa preta mediana longitudinal, processo posterior do escutelo com forte corcova basal e lobo anterior do pronoto sem tubérculos sublaterais. Panstrongylus lignarius foi encontrado em florestas tropicais do Brasil, Suriname, Guiana, Venezuela e Peru. No Brasil está presente nos estados do Amazonas, Maranhão, Tocantins e Pará. Entre 2001 e 2009, foram encontrados cinco espécimes machos de P. lignarius no Estado de Mato Grosso. O primeiro espécime foi encontrado em julho de 2001 no município de Paranaíta (lat. $09^{\circ} 39^{\prime} 53^{\prime \prime}$ Sul e long. $56^{\circ} 28^{\prime} 36^{\prime \prime}$ Oeste), o segundo em março de 2002 no município de Alta Floresta (lat. 09 52' 33” Sul e long. 56 05' 09" Oeste), ambos sem informaçōes do ambiente capturado. Em 2007 foi coletado o terceiro espécime, no intradomicílio, no município de Lucas

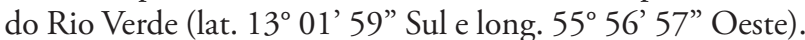
Em setembro de 2009, o quarto espécime de P. lignarius foi capturado por morador entre madeiras empilhadas no peridomicílio, em unidade domiciliar localizada no bairro Novo Horizonte, região periurbana do município de Guarantã do Norte (lat. 0947'15” Sul e long. 5454’36” Oeste). Em novembro de 2009, o último espécime foi encontrado no

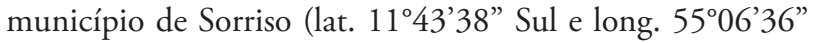
Oeste), próximo a região denominada de Área Verde localizada na área central da cidade, capturado por morador, no peridomicílio (Figura 1). Os municípios de Alta Floresta, Guarantã do Norte e Paranaíta, situam-se no Norte do Estado cuja vegetação é constituída por Floresta Ombrófila Aberta e Densa, Floresta Estacional e Savana, característica de Floresta Amazônica. Os municípios de Lucas do Rio Verde e de Sorriso estão localizados na micro-regiáo geográfica Alto Teles Pires,

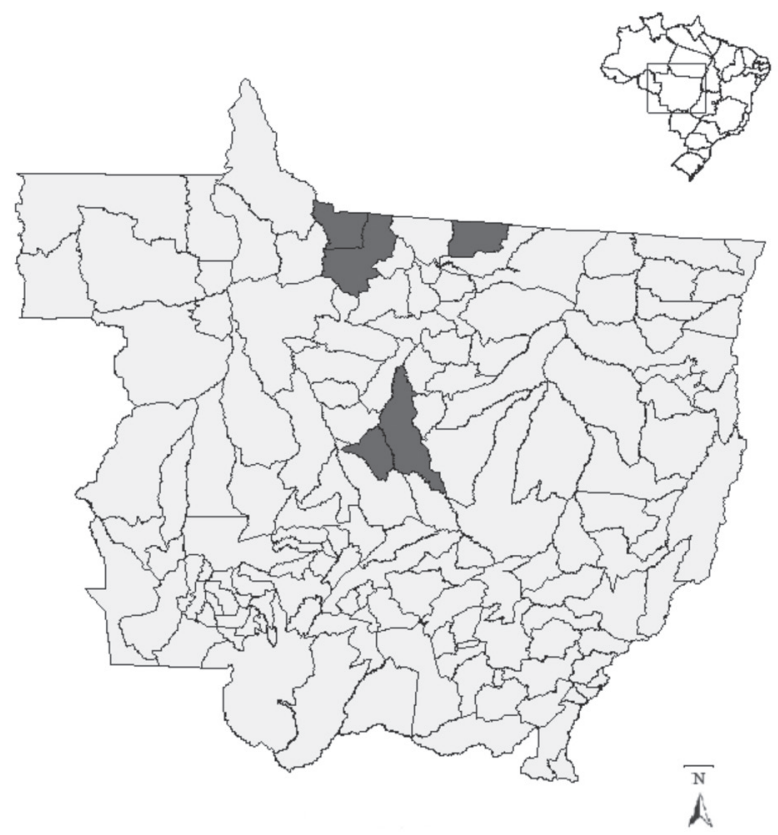

Figura 1 - Municípios de Alta Floresta, Guarantã do Norte e Paranaíta (Norte), Lucas do Rio Verde e Sorriso (Centro), nos quais foram encontrados espécimes de Panstrongylus lignarius no Estado de Mato Grosso, Brasil.

compreendendo áreas de transição entre o Cerrado, Cerradão à Floresta Amazônica, com trechos apresentando vegetaçáo característica de Matas de Galerias (Amaral e Fonzar, 1982). Os municípios têm seu crescimento sócio-econômico, baseado no extrativismo de madeira, produção agrícola, principalmente o plantio de soja, algodáo, milho e pecuária extensiva. $\mathrm{O}$ processo de assentamento familiar realizado nas décadas passadas refletiu positivamente para o estabelecimento da população, entretanto a diminuição na extensão das matas nativas pode estar promovendo a ocorrência esporádica de triatomíneos na proximidade das unidades domiciliares. Lent e Wygodzinsky (1979) mencionaram que espécimes de $P$. lignarius foram encontrados naturalmente infectados pelo Trypanosoma cruzi (Chagas, 1909) porém, não havia na literatura relatos de capturas dentro de moradias no Brasil. Todavia, no Peru P. lignarius, anteriormente denominado 


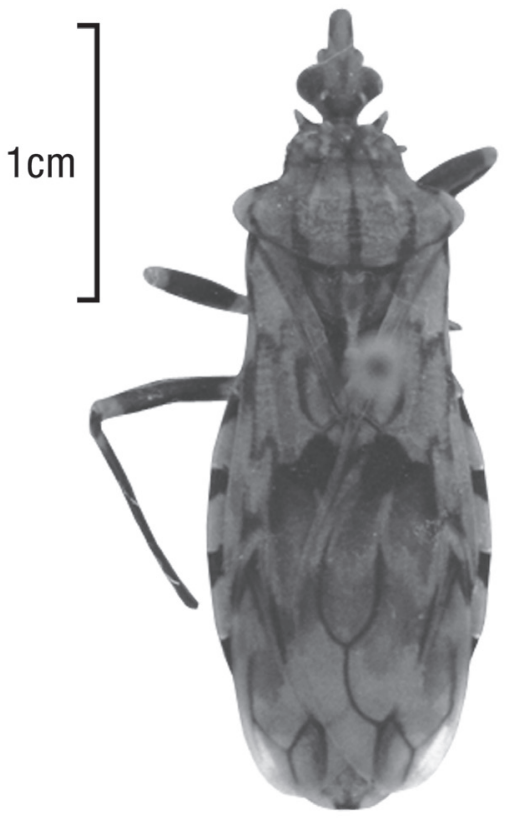

Figura 2 - Vista dorsal do espécime de Panstrongylus lignarius encontrado no município de Paranaíta, Mato Grosso, Brasil.

como $P$. herreri, tem maior índice de domiciliação, sendo considerada a segunda espécie de importância epidemiológica nesse país. Os cinco espécimes de P. lignarius encontrados no Estado de Mato Grosso foram encaminhados para o Laboratório de Entomologia da Secretaria de Estado de Saúde de Mato Grosso (SES-MT). Por estarem mortos e ressecados não foi possível realizar análises parasitológicas para detecção de T. cruzi. Os espécimes estão depositados na Coleçâo Entomológica do Laboratório de Entomologia da SES-MT, sob o registro geral de triatomíneos números 0034; 0043, 0082, 0101 e 0110 respectivamente (Figura 2), onde foram identificados pelos técnicos segundo chave dicotômica de Lent e Wygodzinsky (1979) considerando-se a sinonímia com $P$. herreri. Uma chave dicotômica para as espécies de Panstrongylus mais atualizada foi posteriormente publicada por Bérenguer e Blanchet (2007). Deste modo, o presente trabalho amplia a distribuiçấo geográfica dessa espécie, com a inclusão do Estado de Mato Grosso, Brasil.

\section{AGRADECIMENTOS}

Ao Conselho Nacional de Desenvolvimento Científico e Tecnológico (CNPq), à Secretaria de Vigilância em Saúde MS, à Secretaria de Estado de Saúde de Mato Grosso e à Universidade do Estado de Mato Grosso (UNEMAT) pelo suporte financeiro.

\section{BIBLIOGRAFIA CITADA}

Amaral, D.L.; Fonzar, B.C. 1982. Vegetação. In: BRASIL. Ministério das Minas e Energia. Projeto RADAMBRASIL.Folha SD 21 Cuiabá. Rio de Janeiro, pp.401- 452.

Bérenger, J.M.; Blanchet, D. 2007 A new species of the genus Panstrongylus from French Guiana (Heteroptera; Reduviidae; Triatominae). Memórias do Instituto Oswaldo Cruz, 102: 733-736.

Carcavallo, R.U.; Jurberg, J.; Lent, H. 1999. Filogenia dos triatomíneos, p. 925-969. In: R.U. Carcavallo, I. GalíndezGirón, J. Jurberg, H. Lent (orgs.), Atlas dos vetores da doença de Chagas nas Américas. Editora Fiocruz, Rio de Janeiro, v. III, 1128pp.

Galvão, C.; Carcavallo, R.U.; Rocha, D.S.; Jurberg, J. 2003. A Checklist of the current vali species of the subfamily Triatominae Jeannel, 1919 (Hemiptera, Reduviidae) and their geographical distribuition with nomenclatural and taxonomic notes. Zootaxa, 202: 1-36

Lent, H.; Wygodzinsky, P. 1979. Revision of Triatominae (Hemiptera, Reduviidae), and their significance as vectors of Chagas disease. Bulletin of the American Museum of Natural History 163: 125-520.

Marcilla, A.; Bargues, M.D.; Abad-Franch, F.; Panzera, F; Carcavallo, R.U.; Noireau, F.; Galvão, C.; Jurberg, J.; Miles, M.A.; Dujardin, J.P.; Mas-Coma, S. 2002. Nuclear rDNA ITS-2 reveal polyphyly of Panstrongylus species (Hemiptera: Reduviidae: Triatominae), vectors of Trypanosoma cruzi. Infection, Genetics and Evolution, 1: 225-235.

Santos, C.M.; Jurberg, J.; Galvão, C.; Rocha, D.S.; Fernandez, J.I.R. 2003. Estudo morfométrico do gênero Panstrongylus Berg, 1879 (Hemiptera, Reduviidae, Triatominae) Memórias do Instituto Oswaldo Cruz, 98: 939-944.

Recebido em: 28-11-2011

Aceito em: 19-03-2012 
